\title{
Oncolytic virotherapy for human bone and soft tissue sarcomas using live attenuated poliovirus
}

\author{
SATORU ATSUMI $^{1}$, AKIHIKO MATSUMINE ${ }^{1}$, HIDEMI TOYODA ${ }^{2}$, RUI NIIMI ${ }^{1}$, TAKAHIRO IINO ${ }^{1}$, \\ TOMOKI NAKAMURA $^{1}$, TAKAO MATSUBARA ${ }^{1}$, KUNIHIRO ASANUMA $^{1}$, \\ YOSHIHIRO KOMADA $^{2}$, ATSUMASA UCHIDA ${ }^{1}$ and AKIHIRO SUDO ${ }^{1}$ \\ Departments of ${ }^{1}$ Orthopedic Surgery and ${ }^{2}$ Pediatrics, Mie University \\ Graduate School of Medicine, Tsu, Mie 514-8507, Japan
}

Received March 1, 2012; Accepted May 10, 2012

DOI: $10.3892 /$ ijo.2012.1514

\begin{abstract}
The poliovirus receptor CD155, is essential for poliovirus to infect and induce death in neural cells. Recently, CD155 has been shown to be selectively expressed on certain types of tumor cells originating from the neural crest, including malignant glioma and neuroblastoma. However, the expression pattern of CD155 in soft tissue sarcoma has not been examined. Therefore, we first examined CD155 expression in sarcoma cell lines, and found the expression of both CD155 mRNA and protein in 12 soft and bone tissue sarcoma cell lines. Furthermore, we examined the effect of live attenuated poliovirus (LAPV) on 6 bone and soft tissue sarcoma cell lines in vitro, and found that LAPV induced apoptosis by activating caspases 7 and 3 in all of these cell lines. Furthermore, in BALB/c nu/nu mice xenotransplanted with HT1080 fibrosarcoma cells, administration of live attenuated poliovirus caused growth suppression of the tumors. These results suggest that oncolytic therapy using a LAPV may represent a new option for the treatment of bone and soft tissue sarcomas.
\end{abstract}

\section{Introduction}

The treatment of soft tissue and bone sarcoma remains challenging because higher-grade sarcomas are associated with higher local treatment failure rates and increased metastatic potential. An estimated 10,520 cases of soft tissue sarcoma and 2,650 cases of malignancy of the bones and joints were diagnosed in the United States in 2010 (1). There are more than 50 different histological types of soft tissue sarcoma, and the histological diagnosis of the rare sarcoma is confusing. Typically, low-grade sarcoma demonstrates local invasion with a lower propensity to metastasize, while high-grade sarcomas

Correspondence to: Dr Akihiko Matsumine, Department of Orthopaedic Surgery, Mie University Graduate School of Medicine, 2-174 Edobashi, Tsu, Mie 514-8507, Japan

E-mail: matsumin@clin.medic.mie-u.ac.jp

Key words: oncolytic virotherapy, osteosarcoma, soft tissue sarcoma, poliovirus, CD155/PVR/Necl-5 have a greater likelihood of distant spread to the lungs by a hematogenous route (2).

Surgical resection remains the most effective therapeutic approach for the management of soft tissue sarcoma, especially small (<5 cm), superficial high-grade and low-grade sarcoma (3). Although local control of high-grade soft tissue sarcoma can be obtained through the use of surgery and radiotherapy, recurrence was reported to occur in more than $50 \%$ of such patients (4). In most patients with soft tissue sarcoma that is of a high histological grade, large size $(>5 \mathrm{~cm})$, has invaded deep into the fascia, or is a local recurrence, a combined modality approach comprising preoperative or postoperative chemotherapy is used in addition to the radical surgical procedures (5). However, the role of chemotherapy for adult-type soft tissue sarcoma remains less well defined. According to the results from a meta-analysis of 14 trials performed in 1997 (6), the overall survival at 10 years improved from $50 \%$ to $54 \%$ with adjuvant chemotherapy, but this difference was not statistically significant. Moreover, according to the results from a randomized clinical trial with neoadjuvant chemotherapy (7), the disease-free survival at 5 years improved from $52 \%$ to $56 \%$, but this difference was also not statistically significant. Therefore, the development of a novel therapeutic strategy is required to better treat patients with refractory bone and soft tissue sarcomas.

Oncolytic virotherapy, the selective killing of tumor cells by viruses, is a promising experimental treatment for cancer. The first report of oncolytic virotherapy was due to an unintentional exposure to naturally-occurring viruses or the administration of live attenuated vaccine strains, such as Newcastle disease virus and reovirus (8). Clinical trials of virotherapy for cancer started early in the 20th century. Despite encouraging results in case reports, the overall clinical results have disappointed clinicians because of the weak therapeutic effects if such treatments (9). Recently, a better understanding of the viral tropism for the cells allowed for the development of new strategies to enhance the specificity of viruses for cancer cells and to improve the viral replication in cancer cells (10). In addition to DNA viruses, such as adenovirus and herpes simplex virus, that are molecularly engineered to replicate specifically in tumor cells, RNA viruses with inherent tumor specificity have been developed as oncolytic agents for cancer treatment. This group of viruses includes reovirus (11), Newcastle disease 
virus (12), measles virus (13), vesicular stomatitis virus (14) and poliovirus (15).

The poliovirus is a non-enveloped plus-strand RNA virus belonging to the Picornaviridae, and is the causative agent of paralytic poliomyelitis. The vast majority of poliovirus infections remain asymptomatic, but $1-2 \%$ of cases result in neurologic complications (16). The restriction of poliovirus cell tropism to motor neurons resident within the spinal cord and brainstem gives rise to a highly characteristic clinical syndrome dominated by flaccid paralysis. Selective targeting of motor neurons by poliovirus is most likely determined by the distribution of its cellular receptor, the Ig superfamily molecule CD155 (also known as poliovirus receptor: PVR and nectin-like molecule-5: Necl-5). This assumption is supported by the observation that mice transgenic for human $C D 155$ develop a polio-like syndrome after poliovirus infection (17-19). In addition, intracellular conditions favoring viral replication have also been reported to contribute to poliovirus cell-type specificity (20).

Recently, the biological functions of CD155 have become clearer. It is now known that CD155 plays an important role in cell adhesion, migration, polarization and proliferation (21). CD155 interacts in trans with nectin-3, a member of the Ig-like nectin family, a $\mathrm{Ca}^{2+}$-independent cell-cell adhesion molecule, and cooperatively forms adherence junctions with cadherin. When the cells contact other cells, CD155 is removed from the cell surface by clathrin-dependent endocytosis, due to its trans-interaction with nectin-3. When the cells do not come in contact with other cells, CD155 is upregulated by growth factor-induced signaling, while thus assembling the leading edge of moving cells (22).

Previous reports indicated that malignant tumors originating from the neural crest, including malignant glioma (23) and neuroblastoma (24) could be experimentally treated with various types of live attenuated poliovirus (LAPV), suggested its potential for clinical applications. However, there have been no reports which have so far examined whether bone and soft tissue sarcomas also represent targets of LAPV. In the present study, we first investigated the CD155 expression in bone and soft tissue sarcomas. We next investigated the oncolytic effects of a LAPV on 6 human bone and soft tissue sarcoma cells in vitro. Finally, we examined whether LAPV have oncolytic effects on soft tissue sarcomas using a subcutaneous xenograft animal model.

\section{Materials and methods}

Cell lines. Twelve human bone and soft tissue sarcoma cell lines were used in this study. HT1080 human fibrosarcoma, HS-SY-II human synovial sarcoma, MFH-ino human malignant fibrous histiocytoma, HS-PSS human malignant peripheral nerve sheath tumors (MPNST), HS-Sch-2 human MPNST, NMS-2 human MPNST, 143B human osteosarcoma, Saos-2 human osteosarcoma, and HOS human osteosarcoma cell lines were obtained from Riken Cell Bank (Ibaraki, Japan). HuO9, HuO9-M112, and HuO9-M132 human osteosarcoma cell lines were a kind gift from Dr Yasuo Beppu (National Cancer Center, Tokyo, Japan). HT1080 and 143B cells were maintained in MEM with $10 \%$ fetal bovine serum (FBS) (Invitrogen, Tokyo, Japan). HOS cells were additionally supple- mented with $0.1 \mathrm{mM}$ non-essential amino acids (Invitrogen). HS-SY-II, HS-PSS, HS-Sch-2 cells were maintained in DMEM (Invitrogen) with 10\% FBS. NMS-2, HuO9, HuO9-M112 and HuO9-M132 cells were maintained in RPMI-1640 (Invitrogen) supplemented with $10 \%$ FBS. MFH-ino cells were maintained in DMEM/HamF12 (Invitrogen) with 10\% FBS. Saos-2 cells were maintained in McCoy's (Invitrogen) with 10\% FBS. HeLa and mouse osteosarcoma cell line LM8 cells were grown in MEM and DMEM, respectively, with 10\% FBS.

A live attenuated poliovirus (LAPV). A LAPV vaccine containing the Sabin 1 strain (Japan Poliomyelitis Research Institute, Tokyo, Japan) was used as an oncolytic virus. The virus titer was determined by measuring the $50 \%$ tissue culture infectious dose $\left(\mathrm{TCID}_{50}\right)$ in HeLa cells.

One-step viral growth curves. The growth of poliovirus in the fibrosarcoma cell line, HT1080, was measured as previously described (19). Briefly, cell monolayers were washed with medium, and then were treated with medium containing LAPV at a multiplicity of infection of 0.2 and $2 \mathrm{TCID}_{50}$, respectively. After slowly stirring the dish for $30 \mathrm{~min}$ at room temperature, the cells were thoroughly washed with medium to remove unbound virus, and then incubated in serum-free medium at $37^{\circ} \mathrm{C}$ for different intervals. At 2, 4, 6, 8, 12, 24, and $48 \mathrm{~h}$ after virus inoculation, the extracellular virus and the corresponding cell-associated virus were recovered after three consecutive freeze-thaw cycles. The infectivity of the clarified virus suspension was determined by a $\mathrm{TCID}_{50}$ assay.

Viability assay and morphological features. The effects of LAPV on the viability of sarcoma cells and the morphological changes induced by viral infection were determined. HT1080, MFH-ino, HS-PSS, HuO9-M112, Saos-2, HOS and LM8 cells were seeded at $1.0 \times 10^{4}$ per well on 96 -well plates and treated with LAPV at a multiplicity of infection (MOI) of 2, $0.2,2.0 \times 10^{-2}, 2.0 \times 10^{-3}$ or $2.0 \times 10^{-4} \mathrm{TCID}_{50} /$ cell, respectively. At different intervals, the cell viability was assessed by the MTS assay (CellTiter 96 ${ }^{\circledR}$ AQueous One Solution Cell Proliferation Assay, Promega, Madison, WI, USA). The morphology of cells was evaluated under a phase-contrast microscope.

Apoptosis assay. After $6 \mathrm{~h}$ of exposure to LAPV at a MOI of 2 TCID $_{50} /$ cell or to vehicle, apoptotic HT1080 cells were detected by the TUNEL (terminal deoxynucleotidyltransferase-mediated dUTP-biotin nick end labeling) assay (ApopTag ${ }^{\circledR}$ Peroxidase In Situ Apoptosis Detection Kit, Millipore, Billerica, MA, USA). In addition, the characterization of cell death in HT1080 cells exposed to LAPV was determinated. The HT1080 cells were plated in 96 -well plates at $1.0 \times 10^{4}$ per well. After overnight incubation, the cells were exposed to LAPV at a MOI of 2, 0.2, $2.0 \times 10^{-2} \mathrm{TCID}_{50} /$ cell, or the vehicle for different intervals. After 0 , $6,12,24,36$ or $48 \mathrm{~h}$ of incubation, $20 \mu \mathrm{l}$ of Viability/Cytotoxicity Reagent containing GF-AFC Substrate (ApoTox-Glo ${ }^{\text {TM }}$ Triplex Assay, Promega) was added. After $30 \mathrm{~min}$ of incubation at $37^{\circ} \mathrm{C}$, the fluorescence was recorded at $400 \mathrm{~nm}$ excitation/505 nm emission using a microplate reader for fluorescence and luminescence (Promega) to assess the cell viability. After $100 \mu \mathrm{l}$ of Caspase-Glo 3/7 Regent (ApoTox-Glo Triplex Assay, Promega) was added to the cells and incubated for $30 \mathrm{~min}$ at room tempera- 
ture, the luminescence was recorded. The evaluation of apoptotic cells was performed by measuring the activity of caspases 7 and 3 using a luminogenic substrate. The induction of apoptosis was defined as a decrease in cell viability with a concomitant increase in the caspase 7 and 3 activity.

Total-RNA extraction and quantitative real-time polymerase chain reaction. RNA was isolated (Isogen, Nippon Gene, Tokyo, Japan), reverse transcribed using the 1st Strand cDNA Synthesis Kit (Roche Applied Science, Mannheim, Germany) and subjected to real-time quantitative PCR with ABI PRISM ${ }^{\circledR} 7000$ Sequence Detection System (Applied Biosystems, Carlsbad, CA, USA). Primer are purchased from Applied Biosystems. GAPDH was used as an endogenous 'house-keeping' gene for normalization. Standard curves were generated using cDNA samples from HeLa cells. The relative expression levels of each target gene were indicated by calculating the ratio to those in HeLa cells. All assays were performed in triplicate and repeated three times.

Immunofluorescence microscopy. Cells were fixed with methanol, and blocked with 3\% BSA in PBS. Cells were stained with primary monoclonal antibody against recognizes the poliovirus binding site of CD155 (mouse D171, Neomarkers, Union City, CA, USA) (25), and secondary antibody Alexa ${ }^{\circledR} 488$-conjugated goat anti-mouse $\mathrm{IgG}(\mathrm{H}+\mathrm{L})$ (Invitrogen). The nucleus of each sample was detected by bisBenzimie Hoechst 33342 trihydrochloride staining (Sigma-Aldrich, St. Louis, MO, USA). Microscopic signals were observed with an Olympus BX50 epifluorescence microscope (Tokyo, Japan), and the images were captured with an Olympus DP70 digital camera and processed with an Olympus DPController with the DPManager software program.

Western blot analyses. The expression of CD155 protein was determinated by a western blot analysis as previously described (26). The primary antibody was a goat anti-CD155 antibody, sc-27754 (1:200, Santa Cruz Biotechnology, Santa Cruz, CA, USA) (27), and the secondary antibody reaction was performed using a peroxidase-conjugated secondary antibody (Dako, Carpinteria, CA, USA) and visualized using the ECL substrates (GE Healthcare, Piscataway, NJ, USA).

In vivo xenograft model. Four-week-old BALB/c nu/nu mice were maintained in a humidity- and temperature-controlled laminar flow room. For xenografting, $1 \times 10^{7}$ HT1080 cells in $0.1 \mathrm{ml}$ of PBS were subcutaneously injected into the right flanks of nude mice using a 26-gauge needle. In all the mice, enlargement of the tumors was observed within 1-2 weeks after inoculation. The tumor size was measured with calipers two times a week, and the tumor volume was calculated using the ellipsoid formula: length $\mathrm{x}$ width $^{2} \mathrm{x} 0.52$ (28). When the tumor volume increased to $0.20-0.25 \mathrm{~cm}^{3}$, LAPV $\left(1 \times 10^{6} \mathrm{TCID}_{50}\right)$ or vehicle alone (for control animals) was injected into the right flank tumor once a day for 3 days, and the tumor size was monitored every 3-4 days for 2 weeks.

The mice were sacrificed for histopathological analyses and viral preparation assays. To investigate the histopathological findings, paraffin-embedding samples were observed after hematoxylin and eosin and TUNEL staining. For virus propa- gation assays, tumor samples were immediately frozen and kept at $-80^{\circ} \mathrm{C}$ until use. After homogenization of the tumor samples with additional PBS up to $20 \%$ weight/volume, the tumor tissue homogenates were centrifuged at $2,000 \mathrm{rpm}$ for $20 \mathrm{~min}$ at $4^{\circ} \mathrm{C}$, and the viral titer in the supernatants was determined by the TCID $_{50}$ in HeLa cell culture. All experimental procedures using mice were approved by the Institutional Committees on Animal Welfare.

Statistical analyses. The data were expressed as the means \pm SE. The statistical difference between groups was analyzed by Student's t-test or the Mann-Whitney U test. Differences were considered statistically significant for $\mathrm{p}<0.05$ or $\mathrm{p}<0.01$.

\section{Results}

Expression of CD155 in bone and soft tissue tumor cells. Since CD155 is required for the poliovirus to infect cells, we first examined the expression of $C D 155 \mathrm{mRNA}$ by quantitative realtime PCR. The expressions level of CD155 mRNA in HT1080, MFH-ino, HS-Sch-2, HS-SY-II, HuO9-M112 Saos-2, HOS, and 143B cells were, respectively, 2.3-, 6.7-, 2.5-, 1.9-, 1.7-, 4.4-, 2.4and 5.8-fold higher than those in HeLa cells. In NMS-2, HS-PSS, HuO9 and HuO9-M132 cells, the expression levels of CD155 were almost equal to that of HeLa cells (Fig. 1A).

Next, we confirmed the CD155 expression using an immunofluorescence technique. In all of the human cell lines, CD155 was definitely identified in the cytoplasm and at the intercellular junctions, although the distribution and signal intensity differed among the cell lines (Fig. 1B).

In addition, we performed a western blot analysis to demonstrate the expression level of the CD155 protein. As shown in Fig. 1C, the expression of CD155 protein was observed in all of the bone and soft tissue sarcoma cell lines (molecular weight $\sim 80 \mathrm{kDa})$.

LAPV induces apoptosis in bone and soft tissue sarcoma cells in vitro. To examine whether LAPV induces cell death in sarcoma cell lines, we investigated the morphological changes in the HT1080 cells after LAPV exposure. Under a phase-contrast microscope, the infected HT1080 cells showed morphological changes such as rounding, shrinkage, detachment, and floating in the culture medium within $48 \mathrm{~h}$ (Fig. 2A).

To measure the cell viability after LAPV exposure, 6 human bone and soft tissue tumor cell lines; HT1080, MFH-ino, HS-PSS, HuO9-M112, Saos-2, HOS and one mouse osteosarcoma cell line, LM8, were incubated in the presence of LAPV at a MOI from 2 to $2.0 \times 10^{-4} \mathrm{TCID}_{50} /$ cell and their viability was measured by the MTS assay. LAPV strongly induced cell death in a time- and dose-dependent manner in 5 out of the 6 human bone and soft tissue sarcoma cell lines (Fig. 2B).

At first, the HS-PSS cells seemed to be resistant to LAPV exposure. But, because the HS-PSS cells infected by the LAPV showed morphological changes such as rounding, shrinkage, detachment, and floating in the culture medium, we supposed that the slow growth of the cells minimized the effect of the poliovirus exposure in the MTS assay. Thus, we next observed the effect of LAPV exposure for 7 days, and found that the viability of the HS-PSS cells exposed to the poliovirus was significantly lower than that of the control cells at $72 \mathrm{~h}$ or more 


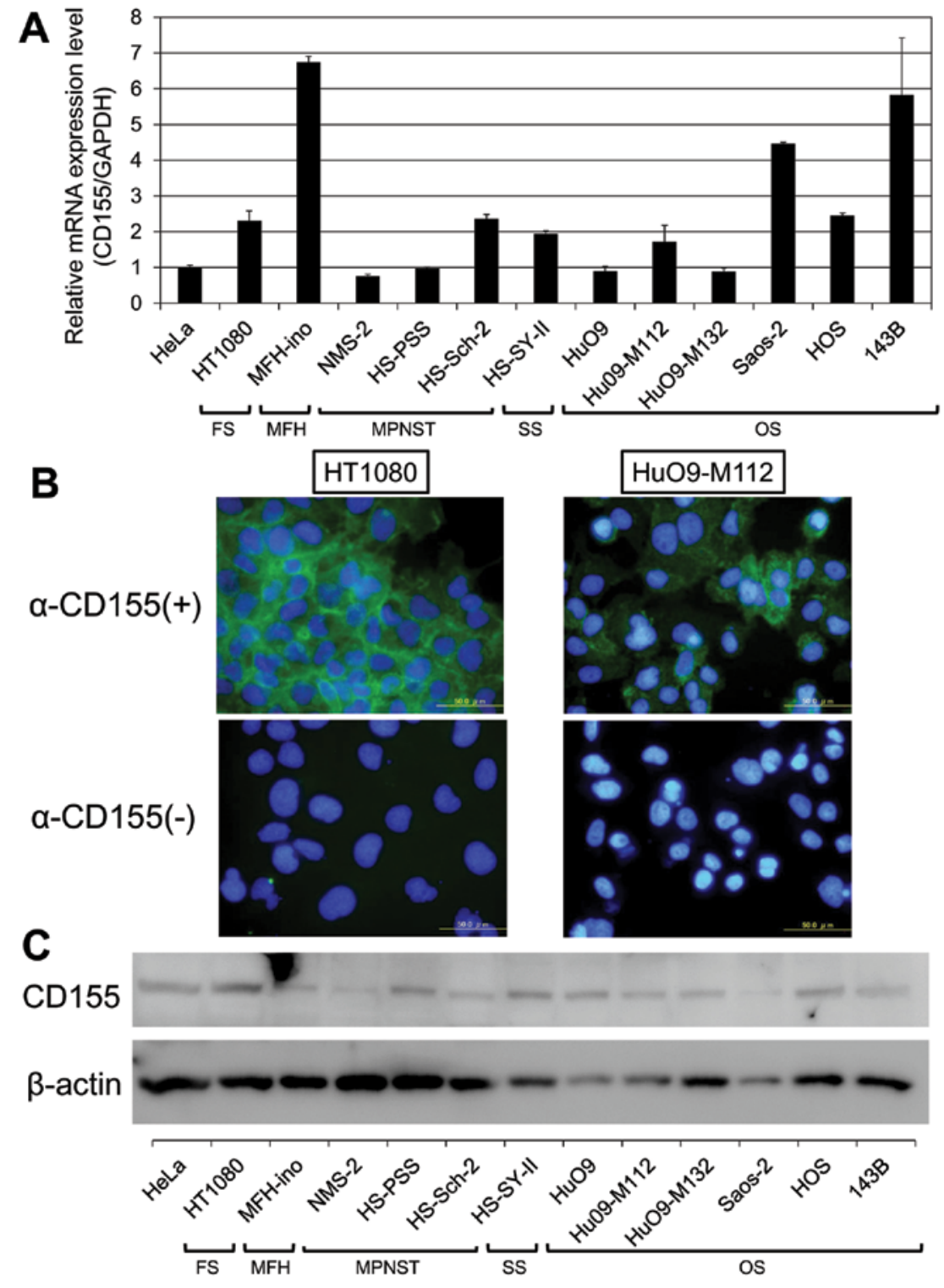

Figure 1. The expression of CD155 in human bone and soft tissue sarcoma cells. (A) The expression of CD155 mRNA in human bone and soft tissue sarcoma cells as determined by quantitative real-time PCR. The relative levels of CD155 mRNA were calculated after normalization with reference to the expression of GAPDH mRNA. The expression levels of CD155 mRNA in HT1080, MFH-ino, HS-Sch-2, HS-SY-II, HuO9-M112 Saos-2, HOS and 143B cells were higher than those in HeLa cells. In the NMS-2, HS-PSS, HuO9 and HuO9-M132 cells, the expression levels of CD155 were almost equal to that of HeLa cells. (B) The immunofluorescence of HT1080 (left) and HuO9-M112 cells (right). CD155 was observed on the cell membrane of these cells. (C) The results of the western blot analysis of CD155 protein expression in human bone and soft tissue sarcoma cells lines. The expression of CD155 protein was observed in all of the bone and soft tissue sarcoma lines that were examined. FS, fibrosarcoma; MFH, malignant fibrous histiocytoma; MPNST, malignant peripheral nerve sheath tumor; SS, synovial sarconma; OS, osteosarcoma.

after infection (data not shown). The viability of the mouse osteosarcoma cell line, LM8, did not differ significantly at any of the time points, and did not exhibit any morphological changes. We considered that the LM8 cell line was completely resistant to LAPV, because mouse cells have no human CD155, which is necessary for the establishment of a poliovirus infection, as was previously known (29).

Our next question was whether the cell growth suppression observed in various sarcoma cell lines was due to apoptotic cell death induced by LAPV. First, we examined the occurrence of apoptotic cell death using the TUNEL assay. We found that the HT1080 cells treated with LAPV had positive staining for the TUNEL reagent (Fig. 3A).
To confirm whether LAPV actually induced apoptotic cell death, the caspase 3/7 activity was measured (Fig. 3C). Our results showed that the poliovirus induced the activation of caspases 3 and 7 in a time- and dose-dependent manner that was consistent with the decrease in cell viability. Hence, we concluded that LAPV induces apoptosis in bone and soft tissue sarcoma cells in vitro.

Propagation of live attenuated poliovirus in soft tissue sarcoma cells. Since LAPV was found to induce apoptosis in the bone and soft tissue sarcoma cells, we next examined the propagation of the poliovirus in sarcoma cells. After exposure to LAPV for $30 \mathrm{~min}$ at a titer of 0.2 and $2 \mathrm{TCID}_{50} /$ cell, the cell-associated 
A

$\operatorname{LAPV}(+)$

LAPV(-)
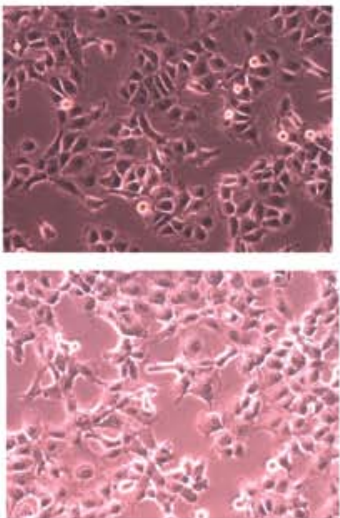

Oh
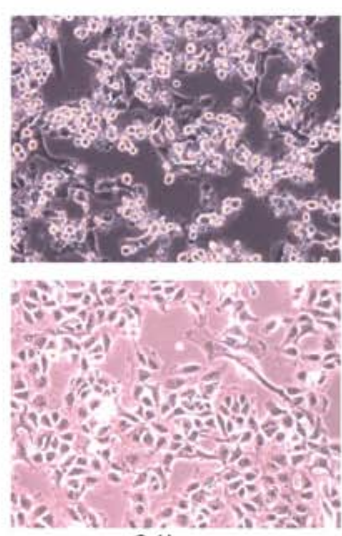

$24 \mathrm{~h}$
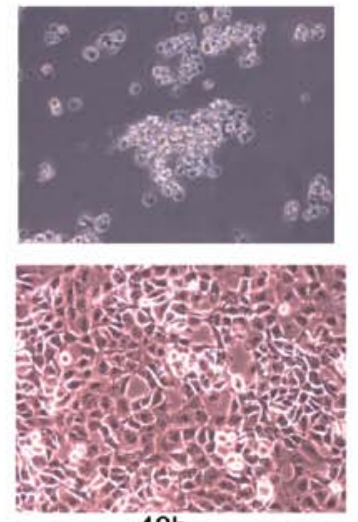

$48 \mathrm{~h}$

Hours after virus infection

B
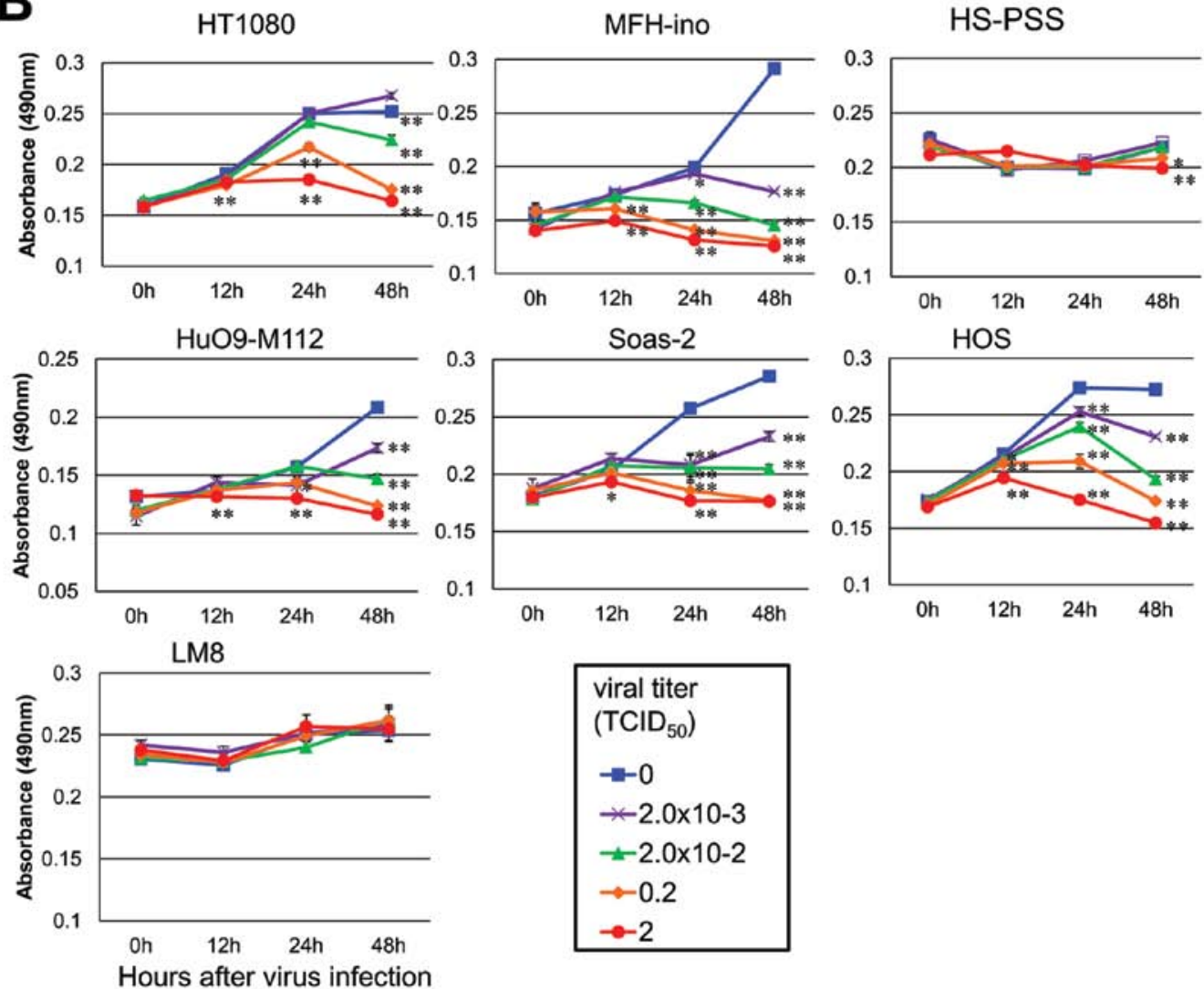

Hours after virus infection

Figure 2. The effect of LAPV. (A) HT1080 cells were photographed before, 24 and $48 \mathrm{~h}$ after LAPV infection. (B) The human fibloblastoma cell line, HT1080, human malignant fibrous histiocytoma cell line, MFH-ino, human MPNST cell line, HS-PSS, human osteosarcoma cell lines, HuO9-M112, Saos-2 and HOS, and the mouse osteosarcoma cell line, LM8, were incubated with LAPV for 12,24 or $48 \mathrm{~h}$ at the indicated MOI $\left(\mathbf{\bullet}, 0 \mathrm{TCID}_{50} ; \mathrm{x}, 2 \times 10^{-3} \mathrm{TCID}_{50} ; \mathbf{\Delta}, 2 \times 10^{-2} \mathrm{TCID}_{50} ;\right.$ $\bullet, 0.2 \mathrm{TCID}_{50} ; \bullet, 2 \mathrm{TCID}_{50}$ ). At different intervals, the cell viability was assessed by the MTS assay. LAPV strongly induced cell death in a time- and dosedependent manner in 5 out of the 6 human bone and soft tissue sarcoma cell lines. The viability of mouse osteosarcoma LM8 cells did not differ significantly at any of the time points. The results are expressed as the means $\pm S E$. ${ }^{*}<<0.05$ and ${ }^{* * *} \mathrm{P}<0.01$ vs. the control (vehicle, 0 TCID $_{50}$ ) group as determined by Student's $t$-test.

and extracellular viral yields were determined by the $\mathrm{TICD}_{50}$ assay. We found that one-step viral growth curves showed the propagation of LAPV in monolayer cultures (Fig. 4).

Live attenuated poliovirus kills soft tissue sarcoma cells in vivo. The inherent capacity for LAPV to kill bone and soft tissue sarcoma cells in vitro suggested that the virus might be therapeutically useful. To test the oncolytic properties of LAPV in vivo, mice bearing subcutaneous HT1080 xenograft tumors were treated with an intratumoral inoculation of LAPV $\left(1 \times 10^{6}\right.$ $\mathrm{TICD}_{50}$ ) once a day for 3 days. The size of the xenotransplants in control mice increased by 16.9 -fold two weeks after treatment. However, three injections of LAPV inhibited the tumor growth by nearly $40 \%$ ( $\mathrm{p}<0.05$ at 7 days or more, Mann-Whitney U test) (Fig. 5). Histopathologically, many TUNEL positive cells were scattered in the xenotransplants on day 1 after treatment with 

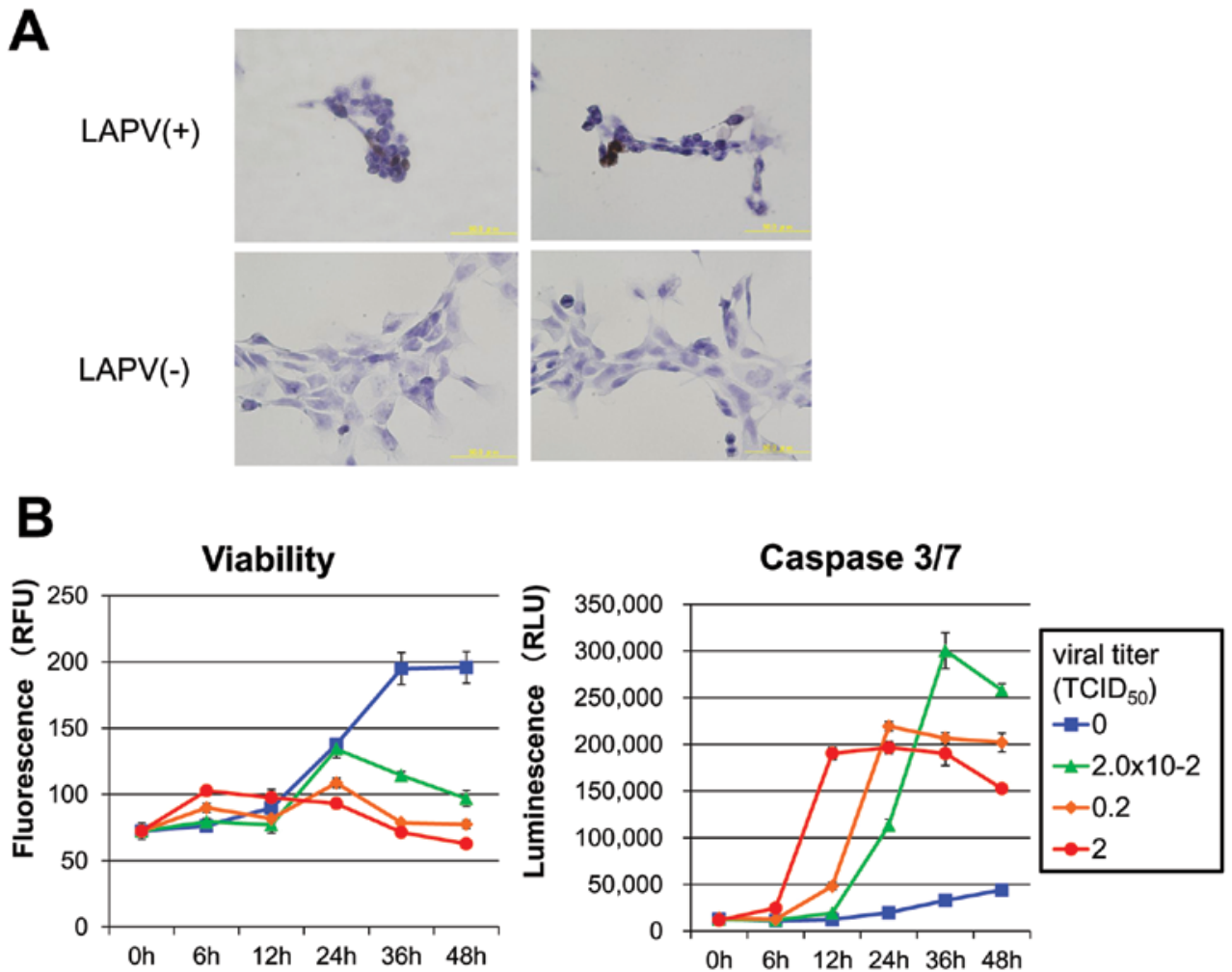

Figure 3. The results of the apoptosis assay in HT1080 cells infected by LAPV. (A) HT1080 cells were incubated with LAPV at a MOI of 2 TCID 50 . Apoptotic HT1080 cells were detected by the TUNEL assay. Positive staining of HT1080 cells was observed after LAPV inoculation (upper panel). (B) The caspase 3/7 activity (right graph) and viability (left graph) of HT1080 cells incubated with LAPV for $6,12,24,36$ or $48 \mathrm{~h}$ at the indicated $\mathrm{MOI}\left(\mathbf{\bullet}, 0 \mathrm{TCID}{ }_{50} ; \mathbf{\Delta}, 2 \times 10^{-2} \mathrm{TCID}_{50} ;\right.$ $\bullet, 0.2 \mathrm{TCID}_{50} ; \bullet, 2 \mathrm{TCID}_{50}$ ) was measured by the ApoTox-Glo Triplex Assay. The poliovirus induced the activation of caspase 3/7 in a time- and dose-dependent manner that was consistent with the decrease in cell viability.
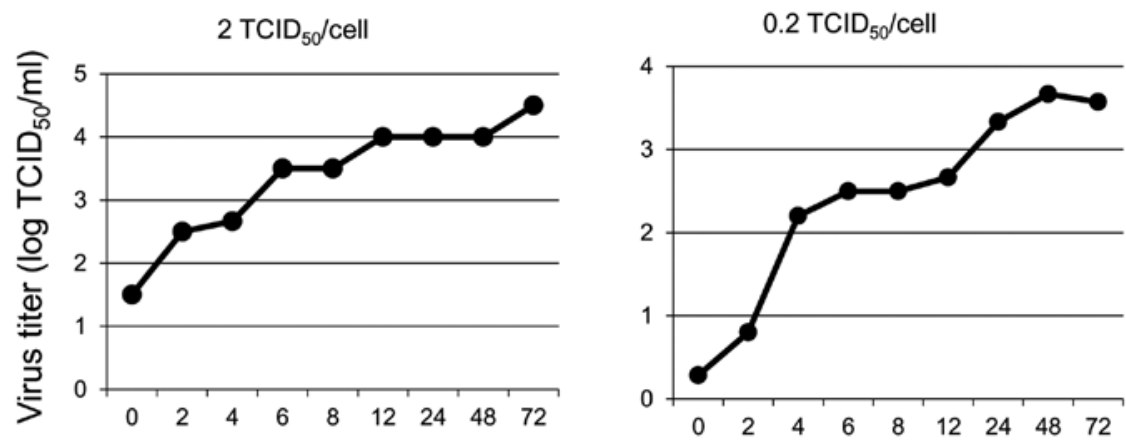

Hours after virus infection

Figure 4. The propagation of LAPV in the HT1080 human fiblosarcoma cell line. One-step growth curves for LAPV were obtained after synchronized infection of monolayer cultures at a MOI of 2 (left graph) and $0.2 \mathrm{TCID}_{50} /$ cell (right graph). LAPV was propagated in HT1080 cells.

LAP, and the areas of TUNEL positive degenerated tissue were obviously increased on day 3 (Fig. 6). TUNEL-positive cells were rarely observed in the xenotransplants treated with the vehicle.

To investigate whether the LAPV is propagated in the xenotransplants, the viral titer was determined using the supernatants of tumor tissue homogenates by measuring the $\mathrm{TCID}_{50}$ in HeLa cell culture. The maximum virus titer was observed on day 3 after inoculation into the tumor (Fig. 7). This indicated that intratumoral replication of LAPV induced the release of a number of infectious viral particles, which lead to the apoptotic cell death of the xenografts.

\section{Discussion}

The clinical outcome of patients with advanced bone and soft tissue sarcomas is still unsatisfactory, despite the use of multidisciplinary treatments including surgery, chemotherapy, and radiotherapy $(5,30)$. Therefore, the development of a novel therapeutic agent is necessary to improve the prognosis of 

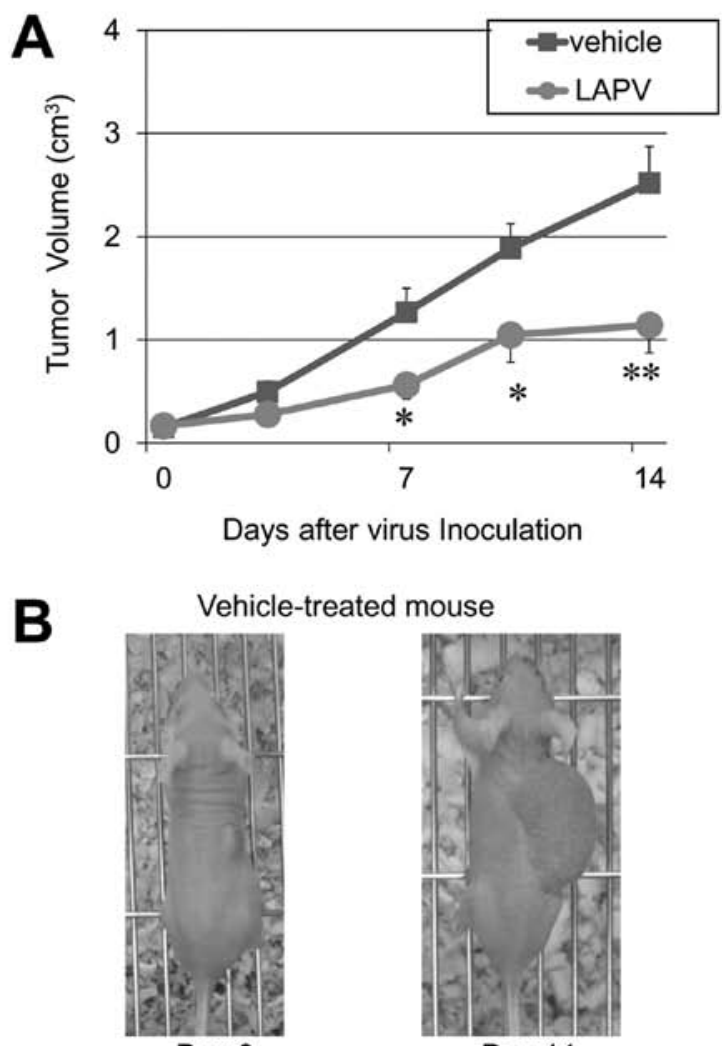

Day 0

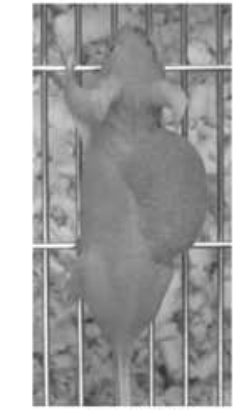

Day 14

LAPV-treated mouse

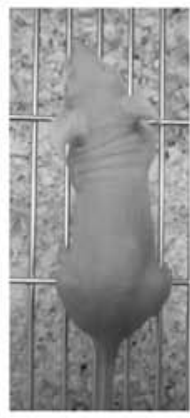

Day 0

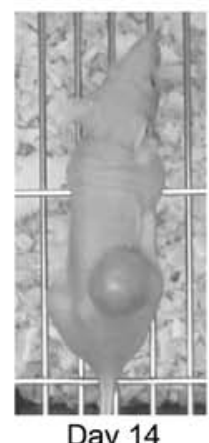

Day 14

Figure 5. The effects of the LAPV on xenografted HT1080 tumors. HT1080 cells were implanted subcutaneously into the right flank of nude mice to form solid tumors. LAPV $(n=14)$ or vehicle $(n=15)$ was injected into right flank tumor once a day for 3 days. (A) The mean volumes of tumors inoculated

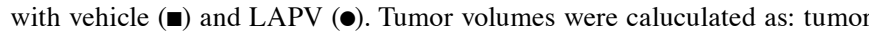
volume $\left(\mathrm{cm}^{3}\right)=0.52 \times \mathrm{a} \mathrm{x} \mathrm{b}^{2}$ (a and $\mathrm{b}$ being the longest and shortest diameters of the tumor, respectively). LAPV injection significantly inhibited the tumor growth compared with vehicle injection from day 7 [data are expressed as the means $\pm \mathrm{SE}$. ${ }^{*} \mathrm{P}<0.05$ and ${ }^{* *} \mathrm{P}<0.01$ vs. the control (vehicle) group as determined by the Mann-Whitney U test]. (B) The growth of the tumors treated with LAPV (upper panel) was significantly suppressed compared to the tumors treated with vehicle (lower panel).

these patients. In this study, we showed that LAPV possess an inherent capacity to induce apoptosis in bone and soft tissue sarcoma cells in vitro and to suppress the growth of implanted fibrosarcoma cells in vivo.

The poliovirus receptor CD155, is a key molecule for oncolytic virotherapy using LAPV, because the expression of CD155 is essential for poliovirus binding and infection (29). We initially supposed that CD155 might be expressed in neurogenic sarcomas, such as MPNST, because the upregulation of CD155 expression was observed in neuroectodermal malignancies (e.g. glioblastoma multiforme, medulloblastoma, or neuroblastoma) (31-33). Surprisingly, however, CD155 expression was observed in all 12 bone and soft tissue sarcoma cell lines examined. This is the first report to show that CD155 is widely expressed in various types of bone and soft tissue sarcoma cell lines. Solecki et al previously showed that the expression of the CD155 gene is transcriptionally activated through the Sonic Hedgehog ( $\mathrm{SHH})$ signaling pathway (34). Recently, activation of the hedgehog signaling pathway was reported in certain sarcoma types $(35,36)$. Upregulated expression of CD155 was demonstrated in NIH3T3 cells transformed by the V12-Ki-Ras oncogene, and it contributes to the loss of contact inhibition in transformed cells (37). Further investigation will be needed to clarify the mechanism of upregulation of CD155 gene expression in sarcoma cells.

We showed that LAPV can kill various types of sarcoma cells, and that there is viral propagation. Gromeier et al have exploited this feature of LAPV to kill glioma cells (15). Poliovirus uniquely depends on CD155 for host cell binding and entry. Based on all available empirical evidence, CD155 is sufficient for all binding and entry functions leading to uncoating of the viral genome $(29,38)$. At first, the HS-PSS cells seemed to be resistant to LAPV exposure. However, the HS-PSS cells infected with the LAPV showed morphological changes such as rounding, shrinkage, detachment, and floating. Since HS-PSS cells have a long cell cycle, we observed the effect of LAPV exposure for 7 days, and confirmed that the HS-PSS cells were also susceptible to LAPV infection. Because of the lack of human type of CD155 expression, the poliovirus could not infect mouse LM8 cells. We concluded that all human bone and soft tissue sarcoma cell lines were susceptible to LAPV infection, and that this was dependent on their CD155 expression.

We showed that the poliovirus infection triggered apoptosis in bone and soft tissue sarcoma cells expressing CD155 by TUNEL staining and the ApoTox-Glo Triplex assay. The ApoTox-Glo Triplex assay showed that the poliovirus induced cell death due to activation of the apoptosis pathway in a time- and dose-dependent manner. In addition, LAPV was propagated in HT1080 cells, as indicated by the one-step viral growth curves analysis. These results suggest that sarcoma cells are targets of the poliovirus. In previous studies, poliovirus infection triggered apoptosis in neuroblastoma cells expressing CD155, as shown by DNA fragmentation, activation of effector caspase activity, and mitochondrial dysfunction $(24,39)$. The replication of poliovirus is restricted to neurons in the spinal cord and brainstem, although CD155 expression is observed in both the target and non-target tissues in humans. This tissue tropism results in a distinct disease pattern unique for poliovirus (40). Ida-Hosonuma et al showed that $\alpha / \beta$ interferon (IFN) determine the tissue tropism by comparing the pathogenesis of the virulent Mahoney strain in CD155-transgenic mice and CD155-transgenic mice deficient in the $\alpha / \beta$ IFN receptor gene (CD155-transgenic/Ifnar knockout mice) (41). CD155-transgenic/Ifnar knockout mice showed increased susceptibility to poliovirus. They subsequently examined the expression of IFN and IFN-stimulated genes (ISGs) in the CD155-transgenic mice. In the non-target tissues, ISGs were expressed even in the non-infected state, and the expression level increased soon after poliovirus infection. On the contrary, 


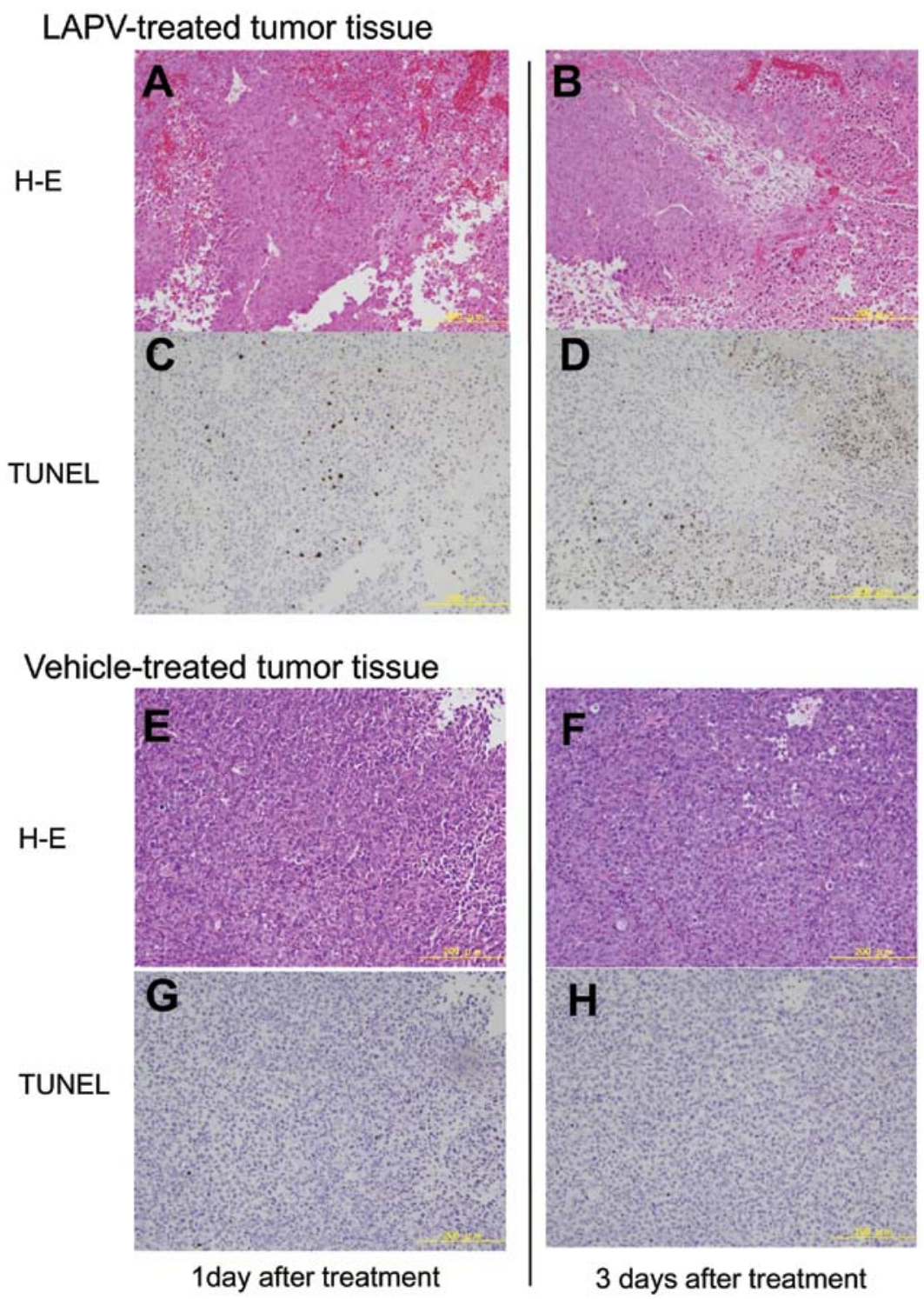

Figure 6. The histopatological evaluation of xenografts after LAPV treatment. TUNEL-positive cells were scattered in the xenotransplants on day 1 after treatment with LAPV, and the areas of TUNEL positive tissue were apparently increased on day 3. (A and C) A subcutaneous tumor treated with LAPV 1 day after treatment (B and D) A tumor treated with LAPV 3 days after the first treatment. ( $\mathrm{E}$ and $\mathrm{G}) \mathrm{A}$ subcutaneous tumor treated with vehicle 1 day after treatment. (F and $\mathrm{H}$ ) A tumor treated with vehicle 3 days after the first treatment. Sections were stained with hematoxylin and eosin (A-B and E-F) or TUNEL (C-D and G-H). Original magnification, $\mathrm{x} 100$.

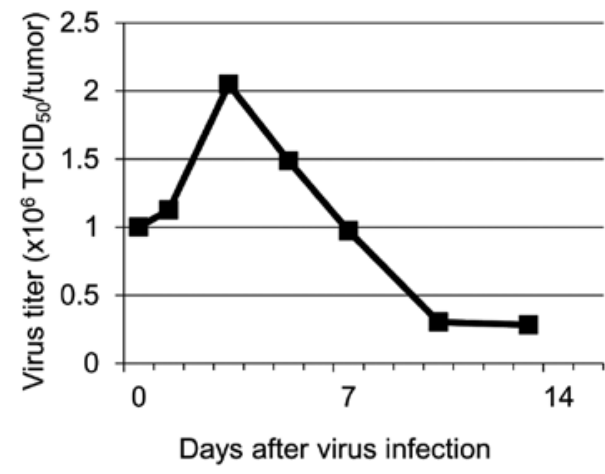

Figure 7. The results of the growth curve analysis of poliovirus in the LAPVtreated tumors. Nude mice bearing subcutaneous HT1080 xenografts were treated with LAPV, euthanized at the indicated intervals, and the viral load in the tumors was measured by the $\mathrm{TCID}_{50}$. The maximum virus titer was observed on day 3 after inoculation of the tumor. in the target tissues, ISG expression was low in the non-infected state and a sufficient response after poliovirus infection was not observed. Although we did not observe the expression of IFN and ISGs in the present study, the low IFN response to the poliovirus may be one of the important determinants of the good susceptibility of sarcoma cells.

We demonstrated that there is efficient oncolysis of subcutaneous xenografted sarcoma in nude mice after intratumoral injection of LAPV. The intratumoral replication of LAPV induced the release of a number of infectious viral particles, which lead to the apoptotic cell death of the xenografts. Therefore, LAPV could be useful for the treatment of bone and soft tissue sarcoma.

The inherent neuropathogenicity of poliovirus is a point of major concern with regard to its potential therapeutic applications. Typically, poliovirus infects the gastrointestinal tract, 
causing mild symptoms or no symptoms at all. In 1 to $2 \%$ of infections, the poliovirus invades the central nervous system, where it uniquely targets motor neurons for destruction, resulting in flaccid paralysis (42). Paralytic poliomyelitis is considered to result from an invasion by circulating poliovirus into the central nervous system, probably via the blood-brain barrier. This notion is supported by a previous study using a mouse model (43). However, an accumulation of patients with vaccine-associated paralytic poliomyelitis following vaccination with orally administered, attenuated poliovirus (OPV) in Romania has been linked to multiple intramuscular (i.m.) injections of various therapeutic or preventive agents administered to OPV recipients (a phenomenon known as 'provocation' poliomyelitis) (44). Recently, the mechanism of provocation poliomyelitis has been proven using poliovirussensitive transgenic mice produced by introducing the human CD155 gene into the mouse genome. Poliovirus particles exist on vesicle structures in the nerve terminals of neuromuscular junctions (45). Skeletal muscle injury induces retrograde axonal transport of poliovirus, and thereby facilitates the viral invasion of the central nervous system and the progression of spinal cord damage (46). In addition, the direct interaction between the cytoplasmic domain of CD155 and Tctex-1 is essential for the efficient retrograde transport of PV-containing vesicles along microtubules in vivo (45).

In our study, LAPV was injected into the right flank tumor once a day for 3 days. It remains unclear whether the intratumoral injection of the LAPV through the muscle surrounding the tumor would be a safe procedure in human subjects. To treat patients with bone and soft tissue sarcomas, it will be necessary to use a highly attenuated phenotype of poliovirus which shows exceedingly poor infectivity in normal neuronal cells while retaining its oncolytic effects against bone and soft tissue sarcomas.

In infected cells, the translation of the plus-strand poliovirus RNA genome is directed by the internal ribosome entry site (IRES), a cis-acting RNA element that facilitates the capindependent binding of ribosomes to an internal site of the viral RNA. In each Sabin vaccine strain, a single point mutation in the IRES secondary-structure domain $\mathrm{V}$ is a major determinant of the neurovirulence attenuation. This point mutation is an A-to-G exchange in the Sabin 1 vaccine strain (A480G according to the Sabin 1 nucleotide numbering) (47). However, because of insufficient genetic stability, the Sabin strain may mutate and convert to a virulent virus during replication in bone and soft tissue sarcomas. To eliminate the neuropathogenic potential, many types of intergeneric poliovirus recombinants were constructed and utilized for the treatment of malignant glioma $(14,15,23)$ and neuroblastoma (48). To treat patients with bone and soft tissue sarcomas, it will be necessary to develop a recombinant poliovirus with a highly attenuated phenotype which minimizes the genetic instability as much as possible.

In the present study, LAPV was injected into the right flank tumor of 4-week-old BALB/c nu/nu mice with an inhibited immune system due to their greatly reduced number of $\mathrm{T}$ cells, which leads to impaired antibody formation that requires $\mathrm{CD} 4^{+}$ helper $\mathrm{T}$ cells. If this treatment strategy is applied to human subjects, the treatment efficacy may be reduced by the neutralizing antibodies against poliovirus which were generated in response to a previous vaccination. However, Toyoda et al (48) showed that neuroblastoma tumors xenografted into CD155 transgenic mice that had previously been immunized with poliovirus still remarkably regressed after intratumoral injection of poliovirus without any side effects. Other previous investigations have also shown that treatment with oncolytic viruses can result in enhancement of the antitumor immune response in vivo $(49,50)$. Thus, we presume that previous poliovirus vaccination might not inhibit the oncolytic effect of LAPV, but actually intensify the oncolysis.

Another potential pitfall is that during a persistent poliovirus infection in a previous study, specific mutations were selected in the first extracellular domain of the CD155 of human neuroblastoma cells, and these mutations increased the resistance of cells to poliovirus-induced lysis (51). The same phenomenon may be observed during the clinical application for bone and soft tissue sarcoma.

In brief, we demonstrated the expression of both CD155 mRNA and protein in bone and soft tissue sarcoma cell lines, and LAPV has oncolytic effects on bone and soft tissue sarcoma cells in vitro and in vivo. The results of our study suggest that LAPV has potential for the clinical treatment of bone and soft tissue sarcomas, but further in vitro and in vivo studies will be required to evaluate the safety of this strategy.

\section{References}

1. Jemal A, Siegel R, Xu J and Ward E: Cancer statistics. CA Cancer J Clin 60: 277-300, 2010.

2. Billingsley KG, Burt ME, Jara E, Ginsberg RJ, Woodruff JM, Leung DH and Brennan MF: Pulmonary metastases from soft tissue sarcoma: analysis of patterns of diseases and postmetastasis survival. Ann Surg 229: 602-610, 1999.

3. Gilbert NF, Cannon CP, Lin PP and Lewis VO: Soft-tissue sarcoma. J Am Acad Orthop Surg 17: 40-47, 2009.

4. Clark MA, Fisher C, Judson I and Thomas JM: Soft-tissue sarcomas in adults. N Engl J Med 353: 701-711, 2005.

5. Grimer R, Judson I, Peake D and Seddon B: Guidelines for the management of soft tissue sarcomas. Sarcoma: May 31, 2010 (Epub ahead of print). doi: 10.1155/2010/506182.

6. No authors listed: Adjuvant chemotherapy for localised resectable soft-tissue sarcoma of adults: meta-analysis of individual data. Sarcoma Meta-analysis Collaboration. Lancet 350: 1647-1654, 1997.

7. Gortzak E, Azzarelli A, Buesa J, et al: A randomised phase II study on neo-adjuvant chemotherapy for 'high-risk' adult softtissue sarcoma. Eur J Cancer 37: 1096-1103, 2001.

8. Sinkovics JG and Horvath JC: New developments in the virus therapy of cancer: a histrogical review. Intervirology 36: 193-214, 1993.

9. Sinkovics JG and Horvath JC: Natural and genetically engineered viral agents for oncolysis and gene therapy of human cancers. Arch Immunol Ther Exp (Warsz) 56 (Suppl 1): 3s-59s, 2008.

10. Parato KA, Senger D, Forsyth PA and Bell JC: Recent progress in the between oncolytic viruses and tumors. Nat Rev Cancer 5: 965-976, 2005

11. Coffey MC, Storong JE, Forsyth PA and Lee PW: Reovirus therapy of tumors with activated Ras pathway. Science 282: 1332-1334, 1998.

12. Sinkovics JG and Horvath JC: Newcastle disease virus (NDV): brief history of its oncolytic strain. J Clin Virol 16: 1-15, 2000.

13. Springfeld C, Fielding AK, Peng KW, Galanis E, Russell SJ and Cattaneo R: Measles virus: improving natural oncolytic properties by genetic engineering. In: Viral Therapy of Human Cancers. Sinkovics JG and Horvath JC (eds). Marcel Dekker Inc., New York, NY, pp459-480, 2005.

14. Stojdl DF, Litchy B, Knowles S, Marius R, Atkins H, Sonenberg N and Bell JC: Exploiting tumor-specific defects in interferon pathway with a previously unknown oncolytic virus. Nat Med 6: 821-825, 2000. 
15. Gromeier M, Lachmann S, Rosenfeld MR, Gutin PH and Wimmer E: Intergenic poliovirus recombinants for treatment of malignant glioma. Proc Natl Acad Sci USA 97: 6803-6808, 2000.

16. Bodian D: Poliomyelitis. In: Pathology of the Nervous System. Minckler J (ed). McGraw-Hill, New York, NY, pp2323-2344, 1972.

17. Ren R, Costantini F, Gorgacz EJ, Lee JJ and Racaniello VR Transgenic mice expressing a human poliovirus receptor: a newmodel for poliomyelitis. Cell 63: 353-362, 1990.

18. Koike S, Taya C, Kurata T, Abe S, Ise I, Yonekawa H and Nomoto A: Transgenic mice susceptible to poliovirus. Proc Natl Acad Sci USA 88: 951-955, 1991.

19. Gromeier M, Alexander L and Wimmer E: Internal ribosomal entry site substitution eliminates neurovirulence in intergenic poliovirus recombinants. Proc Natl Acad Sci USA 93: 2370-2375, 1996.

20. Kauder SE and Racaniello VR: Internal ribosomal entry site substitution eliminates neurovirulence in intergenic poliovirus recombinants. Proc Natl Acad Sci USA 93: 2370-2375, 1996.

21. Takai Y, Miyoshi J, Ikeda W and Ogita H: Nectins and nectinlike molecules: roles in contact inhibition of cell movement and proliferation. Nat Rev Mol Cell Biol 9: 603-615, 2008.

22. Amano H, Ikeda W, Kawano S, et al: Interaction and localization of Necl-5 and PDGF receptor beta at the leading edges of moving NIH3T3 cells: implications for directional cell movement. Genes Cells 13: 269-284, 2008.

23. Merrill MK, Bernhardt G, Sampson JH, Wikstrand CJ, Bigner DD and Gromeier M: Poliovirus receptor CD155targeted oncolysis of glioma. Neuro Oncol 6: 208-217, 2004.

24. Toyoda H, Hayashi T, Gabbazza EC, et al: Experimental treatment of human neuroblastoma using live-attenuated poliovirus. Int J Oncol 24: 49-58, 2004.

25. Nobis P,Zibirre R, Meyer G, Kuhne J, Warnecke G and Koch G: Production of a monoclonal antibody against an epitope on HeLa cells that is the functional poliovirus binding site. J Gen Virol 66: 2563-2569, 1985 .

26. Wakabayashi T, Matsumine A, Nakazora S, et al: Fibulin-3 negatively regulates chondrocyte differentiation. Biochem Biophys Res Commun 391: 1116-1121, 2010.

27. Kono T, Imai Y, Yasuda S, et al: The CD155/poliovirus receptor enhances the proliferation of ras-mutated cells. Int J Cancer 122: 317-324, 2008

28. Tomayko MM and Reynald CP: Determination of subcutaneous tumor size in athymic (nude) mice. Cance Chemother Pharmacol 24: 148-154, 1989.

29. Mendelsohn CL, Wimmer E and Racaniello VR: Cellular receptor for poliovirus: molecular cloning, nucleotide sequence, and expression of a new member of the immunoglobulin superfamily. Cell 56: 855-865, 1989 .

30. Grimer R, Athanasou N, Gerrand C, et al: UK Guidelines for the Management of Bone Sarcomas. Sarcoma: Dec 29, 2010 (Epub ahead of print). doi: 10.1155/2010/317462.

31. Solecki D, Wimmer E, Lipp M and Bernhardt G: Identification and characterization of the cis-acting elements of the human CD155 gene core promoter. J Biol Chem 274: 1791-1800, 1999.

32. Solecki D, Bernhardt G, Lipp M and Wimmer E: Identification of nuclear respiratory factor-1 binding site within the core promoter of polio virus receptor/ CD155 gene. J Biol Chem 275: $12453-12462,2000$

33. Marsson D, Jarry A, Baury B, Blanchardie P, Laboisse C, Lustenberger P and Denis MG: Overexpression of CD155 gene in human colorectal cartinoma. Gut 49: 236-240, 2001.

34. Solecki DJ, Gromeier M, Mueller S, Bernhardt G and Wimmer E: Expression of the human poliovirus receptor/ CD155 gene is activated by sonic hedgehog. J Biol Chem 277: 25697-25702, 2002
35. Li F, Shi W, Capurro M and Filmus J: Glypican-5 stimulates rhabdomyosarcoma cell proliferation by activating Hedgehog signaling. J Cell Biol 192: 691-704, 2011.

36. Oue T, Yoneda A, Uehara S, Yamanaka $\mathrm{H}$ and Fukuzawa $\mathrm{M}$ Increased expression of the hedgehog signaling pathway in pediatric solid malignancies. J Pediatr Surg 45: 387-392, 2010.

37. Minami Y, Ikeda W, Kajita M, Fujito T, Monden M and Takai Y: Involvement of up-regulated Necl-5/Tage4/PVR/CD155 in the loss of contact inhibition in transformed NIH3T3 cells. Biochem Biophys Res Commun 352: 856-860, 2007.

38. Toyoda H, Franco D, Fujita K, Paul AV and Wimmer E: Replication of poliovirus requires binding of the poly $(\mathrm{rC})$ binding protein to cloverleaf as well as to the adjacent C-rich spacer sequence between the cloverleaf and the internal ribosomal entry site. J Virol 81: 10017-10028, 2007.

39. Gosselin AS, Simonin Y, Guivel-Benhassine F, et al: Poliovirusinduced apoptosis is reduced in cell expressing a mutant CD155 selected during presistant poliovirus infection in neuroblastoma cells. J Virol 77: 790-798, 2003.

40. Whitton JL, Cornell CT and Feuer R: Host and virus determinants of picornavirus pathogenesis and tropism. Nat Rev Microbiol 3: 765-776, 2005.

41. Ida-Hosonuma M, Iwasaki T, Yoshikawa T, et al: The alpha/ beta interferon response controls tissue tropism and pathogenicity of poliovirus. J Virol 79: 4460-4469, 2005.

42. Melnick JL: Enteroviruses: polioviruses, coxsackieviruses, echoviruses, and newer enteroviruses. In: Virology. Fields BN, Knipe DM, Howley PM, et al (eds). Raven Press, New York, NY, pp549-605, 1995

43. Yang WX, Terasaki T, Shiroki K, et al: Efficient delivery of circulating poliovirus to the central nervous system independently of poliovirus receptor. Virology 229: 421-428, 1997.

44. Strebel PM, Ion-Nedelcu N, Baughman AL, Sutter RM and Cochi SL: Intramuscular injections within 30 days of immunization with oral poliovirus vaccine - a risk factor for vaccine-associated paralytic poliomyelitis. N Engl J Med 332: 500-506, 1995

45. Ohka S, Matsuda N, Tohyama K, Oda T, Morikawa M, Kuge S and Nomoto A: Receptor(CD155)-dependent endocytosis of poliovirus and retrograde axonal transport of the endosome. J Virol 78: 7186-7198, 2004.

46. Gromeier M and Wimmer E: Mechanism of injury-provoked poliomyelitis. J Virol 72: 5056-5060, 1998.

47. Ochs K, Zeller A, Saleh L, Bassili G, Song Y, Sonntag A and Niepmann M: Impaired binding of standard initiation factors mediates poliovirus transla tion attenuation. J Virol 77: 115-122, 2003.

48. Toyoda H, Yin J, Mueller S, Wimmer E and Cello J: Oncolytic treatment and care of neuroblastoma by a novel attenuated poliovirus in a novel poliovirus-susceptible animal model. Cancer Res 67: 2857-2864, 2007.

49. Porosnicu M, Mian A and Barber GN: The oncolytic effect of recombinant vesicular stomatitis virus in enhanced by expression of the fusion cytosine deaminase/uracil phosphoribosyltransferase suicide gene. Cancer Res 63: 8366-8376, 2003 .

50. Obuchi M, Fernandez M and Barber GN: Development of recombinant vesicular stomatitis virus that exploit defects in host defense to augment specific oncolytic activity. J Virol 77: 8843-8856, 2003.

51. Pavio N, Couderc T, Girard S, Sgro JY, Blondel B and ColbèreGarapin F: Expression of mutated poliovirus receptors in human neuroblastoma cells persistently infected with poliovirus. Virology 274: 331-342, 2000 